\title{
Appréciation simple de la maturité des composts urbains en relation avec leurs effets sur la pro- duction végétale
}

Bernard NICOLARDOT, Rémi CHAUSSOD, Jean-Louis MOREL $\left({ }^{*}\right)$, Armand GUCKERT $\left({ }^{*}\right)$, Daniel BENISTANT, Gérard CATROUX \& Jean-Claude GERMON

I.N.R.A., Laboratoire de Microbiologie des Sols, Centre de Recherches de Dijon, I7, rue Sully, B.P. 1540, F 21034 Dijon Cedex

(*) E.N.S.A.I.A., 2, avenue de la Forêt de Haye, F 54500 Vandouvre

Des composts prélevés dans différentes usines après divers temps de maturation ont été éprouvés sur végétaux (laitue, ray-grass). Par rapport à la quantité de matière sèche produite par le ray-grass sur le sol seul, on observe un effet dépressif marqué lorsque le sol est additionné de composts jeunes, puis cet effet s'atténue et devient même positif après plusieurs mois de maturation. Des observations identiques ont été effectuées sur laitue avec les produits d'une même usine : la production de matière sèche et la quantité d 'azote exportée augmentent avec le temps de maturation.

L'étroite corrélation existant entre les valeurs de consommation d'oxygène par les composts et les rendements en matière sèche permet de mettre en relation la maturité et les caractéristiques biologiques ou physico-chimiques des composts urbains. Ces expériences autorisent en outre la définition de seuils de maturité.

Ces études ont conduit les auteurs à proposer deux tests simples de détermination de la maturité :

- une méthode respirométrique qui nécessite un matériel courant, un délai de 3 à $7 \mathrm{j}$ et $1 \mathrm{~kg}$ de produit frais. Les résultats obtenus avec cette technique concordent parfaitement avec ceux du laboratoire ;

- une méthode physico-chimique où sont dosés les composés carbonés facilement fermentescibles. Le taux de minéralisation globale du carbone, "T.M.G. 21 », qui reflète le degré de stabilisation atteint par les composts est en effet relié au taux de carbone organique total et à la qualité des sucres solubles présents.

Finalement, l'association de la mesure de la consommation d'oxygène d'un compost et de la détermination de son « T.M.G. 21 » paraît souhaitable, évitant ainsi les biais liés à l'une ou l'autre des 2 méthodes.

Mots clés additionnels : Effet dépressif, biodégradabilité, polysaccharides, respirométrie, méthode de mesure. yield.

Samples of compost were taken from several composting plants after various times of maturation and were tested on lettuce and rye-grass. A marked depressive effect was observed on the yield of rye-grass grown on soil amended with raw compost compared to control soil ; this effect then vanished and even became positive after several months of maturation. With composts from the same origin, similar observations were made on lettuce : the dry matter and the amount of nitrogen exported increased with the length of maturation. The close correlation between oxygen consumption by composts and dry matter yield made it possible to establish relationships between maturity and biological or physico-chemical characteristics of composts. Moreover, these experiments led to the definition of maturity levels. From this work, two simple methods for the determination of maturity are suggested : 1) a respirometric method which requires an ordinary vessel, a 3-7 days incubation period and one kilogram of fresh compost sample. The results obtained with this method were in good agreement with laboratory studies ; 2) a physico-chemical method in which easily degradable carbon compounds are analysed. The overall mineralization rate of carbon «T.M.G. $21 »$, which reflects the degree of stabilization of composts, was in fact related to the total organic content and the quality of soluble carbohydrates in the compost. Finally, it seems desirable to combine the oxygen consumption measurement and compost « T.M.G. 21 » determination to eliminate any bias induced by one or the other method.

Additional key words : Depressive effect, biodegradability, polysaccharides, respirometry, methodology. 


\section{INTRODUCTION}

L'utilisation des composts urbains en agriculture peut occasionnellement entraîner des effets dépressifs sur la végétation dont la cause semble être la présence de produits phytotoxiques (TROCMÉ \& BONIFACE, 1977 ; JUSTE et al., 1980) ou, beaucoup plus souvent, l'insuffisance de maturité. Cette dernière se traduit généralement par une carence en azote (POMMEL \& JUSTE, 1977) ou par des signes de toxicité liés à une anaérobiose ou à un échauffement consécutifs à la présence de produits facilement fermentescibles.

En France, la norme AFNOR NFU 44051 (1981) définit un compost mûr comme étant un mélange de produits qui a subi une fermentation thermophile suivie d'une maturation. En fait, nous admettrons qu'un compost est mûr lorsqu'il n'exerce plus d'effets dépressifs sur la végétation aux doses normales d'application.

La méthode de référence pour apprécier la maturité d'un compost apparaît donc être l'utilisation d'essais sur végétaux qui sont cependant des approches expérimentales longues et lourdes. Aussi, des techniques plus simples ont-elles été proposées. Elles reposent sur différents critères :

- inhibition de germination sur cresson (SPOHN, 1968) ou sur orge et haricots nains (HIRSCHEYDT, 1977) ;

- observations de phénomènes physiques : capacité d'autoéchauffement (SPOHN, 1968) ou faculté de tassement (JUSTE \& SOLDA, 1977) ;

- mesures physico-chimiques ou chimiques portant sur le pH, le rH (MOLLER, 1968), les matières organiques dégradables (ROLLE \& ORSANIC, 1964), la demande chimique en oxygène (Lossin, 1971), la caractérisation des sulfures ou des différentes formes d'azote minéral (SPOHN, 1968, 1978), la présence d'amidon (Lossin, 1970) et le rapport C/N (HIRSCHEYDT, 1977), ou encore la chromatographie circulaire (HeRTElENDy, 1974) et la mesure de la densité optique d'un extrait alcalin de compost (MOREL, 1982 ; BIDLINGMAIER, 1984) ;

- mesures de l'activité biologique par dénombrement de la population microbienne (AHRENS et al., 1965), dosage de l'ATP (Colin, 1977), étude de la croissance en boîte de Petri de différents champignons sur milieu contenant du compost (OBRIST, 1965 ; CHROMETZKA, 1968a), mesure du dégagement de $\mathrm{CO}_{2}$ (SPOHN \& KNEER, 1968) ou de la consommation d'oxygène à l'aide de l'appareil de WARBURG (CHROMETZKA, 1968 $b$; NIESE, 1969) ou d'un appareil mieux adapté (ALLENSPACH, 1969).

Malheureusement, parmi tous les travaux présentés, peu d'auteurs ont eu le souci de confronter leurs résultats avec ceux d'une croissance de végétaux.

L'objectif de notre étude sera double :

- Il ne s'agira pas de porter un jugement global sur la valeur agronomique des composts mais, plus simplement, "l'effet dépressif 》 étant généralement attribué à une maturité insuffisante, nous chercherons à mettre en évidence d'éventuelles relations entre l'état de maturité du compost et ses effets immédiats sur la croissance des plantes, incluant aussi bien l'effet azote qu'une éventuelle phytotoxicité. Dans ce but, nous comparerons les réponses de 2 plantes très différentes
- la laitue et le ray-grass - à un même compost pris à différents états de maturité, puis, en conservant le ray-grass comme plante-test, nous analyserons les effets de composts provenant de 6 usines différentes.

- Nous proposerons également des techniques d'estimation de la maturité qui soient d'un usage simple pour pouvoir être utilisées facilement en usine et dont les résultats auront été vérifiés par des tests de végétation. Nos expériences préliminaires (BENISTANT, 1978) nous ont conduits à poser comme hypothèse de départ qu'un compost est d'autant plus mûr que ses composés fermentescibles ont été mieux biodégradés et donc que son activité respiratoire est plus réduite. Deux approches seront donc décrites dans ce travail : une méthode respirométrique et une méthode physico-chimique qui fait intervenir le dosage de certaines matières organiques fermentescibles des composts.

\section{MATÉRIELS ET MÉTHODES}

\section{A. Matériel d'étude}

\section{Composts}

Les caractéristiques analytiques des composts utilisés ainsi que les procédures de prélèvement et de préparation des échantillons sont décrits par MOREL et al. (1986). Nous adopterons la même dénomination proposée par ces auteurs pour les différentes usines d'où proviennent les produits étudiés.

\section{Sol}

Sol brun calcique de la région de Dijon (argiles/ limons $/$ sables $=33 / 54 / 13$ p. $100 ;$ carbone $=$ 1,32 p. $100 ;$ azote total $=1,55$ p. $1000 ; \mathrm{CaCO}_{3}=$ $1,3$ p. $100 ; \mathrm{pH}$ eau $=7,6)$.

\section{B. Culture de végétaux}

\section{Comparaison laitue - ray-grass (expérience 1)}

Cette expérience met en œuvre le compost de l'usine $A$ prélevé après $4,60,120,180$ et $240 \mathrm{j}$ de maturation et apporté à différentes doses dans le sol.

Les graines de Lactuca sativa var. "Norand" (J. L.) sont mises à germer sur vermiculite en salle climatisée. Au stade 5 feuilles, une plantule est repiquée dans chacun des pots d'essais contenant $1,7 \mathrm{~kg}$ de terre additionnée ou non de compost aux doses de 12, 24,48 et $96 \mathrm{~g}$ de compost $/ \mathrm{kg}$ de sol soit $30,60,120$ et $240 \mathrm{t} / \mathrm{ha}$. En plus du témoin sans compost (sol seul), un traitement «fertilisation minérale N-P-K » est mis en place ; 4 répétitions sont effectuées pour chacun des traitements, les pots étant disposés en 4 blocs randomisés (22 pots par bloc). La culture en serre froide (température entre 13 et $19^{\circ} \mathrm{C}$ ) avec éclairage d'appoint dure 10 semaines. L'humidité des pots est périodiquement ramenée par pesée à une valeur correspondant à la capacité de rétention. Après récolte, les parties aériennes sont séchées à $105^{\circ} \mathrm{C}$ et pesées. 
100 graines de Lolium italicum var. "Sérénade 》 (tétraploïde) sont semées dans des pots contenant $200 \mathrm{~g}$ de sol additionné ou non de compost aux doses de 24 et $96 \mathrm{~g} / \mathrm{kg}$ (60 et $240 \mathrm{t} / \mathrm{ha}$ ). Chaque traitement est répété 4 fois et les pots sont rassemblés en 4 blocs randomisés (11 pots par bloc) dans une salle climatisée : température diurne $25^{\circ} \mathrm{C}$, température nocturne $20^{\circ} \mathrm{C}$, éclairage $16 \mathrm{~h}$ par jour à raison de $247 \mu \mathrm{E} . \mathrm{s}^{-1} \cdot \mathrm{m}^{-2}$. L'humidité du sol est contrôlée par pesée et maintenue à 80 p. 100 de la capacité de rétention par des arrosages quotidiens. La coupe est effectuée après 4 semaines et les parties aériennes sont séchées à $105^{\circ} \mathrm{C}$, puis pesées.

2. Test sur ray-grass avec divers composts (expérience 2)

Les produits proviennent des usines B, C, D, E, I, où ils ont été prélevés à différents stades de maturation. Ils sont ici mélangés au sol à raison de $24 \mathrm{~g}$ de compost $/ \mathrm{kg}$ de terre (dose équivalente à $60 \mathrm{t} / \mathrm{ha}$ de matière sèche). Une culture de ray-grass est effectuée dans des conditions identiques à celles décrites précédemment, les seules différences étant l'apport d'une solution nutritive (9 mg $\mathrm{N} ; 3,9 \mathrm{mg} \mathrm{P} ; 7,4 \mathrm{mg} \mathrm{K}$ ) dans chaque pot lors du semis et la récolte d'une seule coupe, après 5 semaines.

\section{Mesures respirométriques}

Elles sont effectuées à l'aide des respiromètres électrolytiques décrits par GERMON \& VERGUET (1974). Le mode opératoire a été donné antérieurement (MOREL et al., 1986).

\section{Test respirométrique simple}

La méthode proposée repose sur l'estimation de la consommation d'oxygène du compost à partir de la mesure de la dépression créée lors de l'incubation d'un échantillon dans une enceinte étanche, le $\mathrm{CO}_{2}$ produit étant piégé par de la soude. L'enceinte d'incubation consiste en un auto-cuiseur ménager (S.E.B.) de $23,9 \mathrm{l}$, relié par un tube à vide à un tensiographe (Fournier-Euvrard) d'un temps de révolution de $7 \mathrm{j}$ et étalonné pour mesurer des dépressions comprises entre 0 et $20 \mathrm{~cm}$ de mercure.

Les mesures sont réalisées sur $1 \mathrm{~kg}$ de compost frais, de même origine que les compost traités en respiromètres électrolytiques. Pour certains composts jugés trop secs lors de leur prélèvement, l'humidité est amenée à environ 50 p. 100 avant la mise en incubation. L'incubation est effectuée pendant 3 j à $20^{\circ} \mathrm{C}$ avec 2 répétitions, le $\mathrm{CO}_{2}$ dégagé étant piégé par $250 \mathrm{ml}$ de $\mathrm{NaOH} 2 \mathrm{~N}$. La consommation d'O $\mathrm{d}_{2} \mathrm{du}$ compost est calculée à l'aide de la formule suivante :

\section{Consommation}

moyenne horaire

d'oxygène

$(\mathrm{mg} \mathrm{O} / \mathrm{kg} / \mathrm{h})$

$=\mathrm{V} \times \frac{\Delta \mathrm{P}}{76} \times \frac{32}{24,04} \times \frac{1000}{\mathrm{Pc}} \times \frac{1000}{\mathrm{t}}$
V : Volume gazeux $=$ Volume de l'enceinte Volume du compost - Volume de la soude (en l),

$\Delta \mathrm{P} \quad$ : Dépression maximum atteinte en $3 \mathrm{j}$ (en $\mathrm{cm}$ de mercure),

Pc : Poids de compost sec utilisé (en g),

$\mathrm{t}$ : Temps de consommation $\mathrm{d}^{\prime} \mathrm{O}_{2}$ ou temps nécessaire pour parvenir à la dépression maximum atteinte, diminué du temps de latence (en h),

24,04: Volume d'une mole d' $\mathrm{O}_{2}$ à $20^{\circ} \mathrm{C}$ et sous $76 \mathrm{~cm}$ de mercure (en l),

32 : Poids d'une mole d' $\mathrm{O}_{2}$ (en g),

76 : Pression atmosphérique (en $\mathrm{cm}$ de mercure). On suppose que la pression atmosphérique externe est toujours de $76 \mathrm{~cm}$ de mercure et on néglige les erreurs sur les volumes liées à des pressions atmosphériques initiales différentes. Le volume du compost est apprécié dans chaque cas en fin d'incubation par tassement du produit dans un récipient gradué.

\section{E. Test de maturité effectué par la caractérisation de composés biodégradables}

MOREL et al. (1986) ont défini un indice de biodégradation à partir du taux de minéralisation globale du carbone à 21 j (T.M.G. 21) qui correspond à la quantité de carbone minéralisé sous forme $\mathrm{CO}_{2}$ par un mélange sol + compost pendant $21 \mathrm{j}$ d'incubation, exprimée en pourcentage du carbone initial total de ce mélange. Cet indice reflète l'activité minéralisatrice d'un sol donné, en présence de divers produits, et rend ainsi compte de la stabilité biologique atteinte par le compost.

\section{RÉSULTATS - DISCUSSION}

\section{A. Effets de la maturation des composts sur la pro- duction de matière sèche végétale}

\section{Expérience 1 : Comparaison laitue - ray-grass}

On constate (tabl. 1) que les réactions, à un même compost, de 2 plantes aussi différentes que peuvent l'être une dicotylédone maraîchère (la laitue) et une monocotylédone fourragère (le ray-grass), sont tout à fait comparables. Aussi bien pour la laitue que pour le ray-grass, l'analyse statistique des rendements met en évidence une interaction entre la dose et l'âge du compost, ce dernier facteur ayant une forte influence. Les composts âgés de $4 \mathrm{j}, 60 \mathrm{j}$ et $120 \mathrm{j}$ ont des effets dépressifs marqués sur la laitue comme sur le raygrass et ce d'autant plus que la dose est plus élevée. L'effet dépressif disparaît après $120 \mathrm{j}$, car pour les composts de $180 \mathrm{j}$ les rendements en matière sèche sont égaux ou supérieurs aux témoins : pour le raygrass, le rendement est significativement supérieur aux témoins "sol seul » lorsque le compost de $180 \mathrm{j}$ est apporté à la dose de $240 \mathrm{t} /$ ha ; pour la laitue, l'effet est significativement positif avec le compost âgé de $240 \mathrm{j}$ aux 2 plus fortes doses. Les rendements sont 
TABLEAU 1

Rendements en matière sèche $(\mathrm{g} / \mathrm{pot})$ de laitue et de ray-grass (expérience $\mathrm{l})$. Dry matter yields (g/pot) of lettuce and rye-grass (experiment l).

\begin{tabular}{|c|c|c|c|c|c|}
\hline $\begin{array}{l}\text { Age du compost } \\
\text { (en jours) }\end{array}$ & 4 & 60 & 120 & 180 & 240 \\
\hline \multicolumn{6}{|c|}{ Caractéristiques du compost Aa (*) } \\
\hline $\mathrm{C} \ll$ Anne $» \%$ & 33,7 & 30,4 & 23,7 & 16,8 & 14,3 \\
\hline$N \ll$ Kjeldahl $» \%_{0}$ & 0,98 & 0,96 & 1,19 & 1,10 & $\mathbf{1}, 19$ \\
\hline $\mathrm{C} / \mathrm{N}$ & 34,4 & 31,7 & 19,9 & 15,3 & 12 \\
\hline \multicolumn{6}{|l|}{ Laitue } \\
\hline Témoin $=\begin{array}{l}3,430 \mathrm{e} \\
(43,4)\end{array}$ & & & & & \\
\hline \multicolumn{6}{|l|}{ Témoin + NPK $=\underset{(108,9)}{4,572 f g}$} \\
\hline $30 \mathrm{t} / \mathrm{ha}$ & $\begin{array}{c}0,920 \text { bc } \\
(13,5)\end{array}$ & $\begin{array}{l}0,656 \mathrm{ab} \\
(6,9)\end{array}$ & $\begin{array}{c}2,594 \mathrm{~d} \\
(29,1)\end{array}$ & $\begin{array}{l}3,562 \mathrm{e} \\
(50,2)\end{array}$ & $\begin{array}{l}3,212 \mathrm{e} \\
(45,3)\end{array}$ \\
\hline $60 \mathrm{t} / \mathrm{ha}$ & $\begin{array}{c}0,167 \mathrm{ab} \\
(4,9)\end{array}$ & $\begin{array}{c}0,083 \text { a } \\
(1,4)\end{array}$ & $\begin{array}{l}1,449 \mathrm{c} \\
(15,8)\end{array}$ & $\begin{array}{l}3,427 \mathrm{e} \\
(51,7)\end{array}$ & $\begin{array}{l}3,665 \mathrm{e} \\
(55,1)\end{array}$ \\
\hline 120 tha & $\begin{array}{c}0,100 \mathrm{a} \\
(3,5)\end{array}$ & $\begin{array}{l}0,043 \text { a } \\
(\mathrm{ND})\end{array}$ & $\begin{array}{c}0,526 \text { ab } \\
(9,2)\end{array}$ & $\begin{array}{l}3,988 \text { ef } \\
(89,4)\end{array}$ & $\begin{array}{l}4,582 \mathrm{fg} \\
(72,2)\end{array}$ \\
\hline $240 \mathrm{t} / \mathrm{ha}$ & $\begin{array}{l}0,035 \mathrm{a} \\
\text { (ND) }\end{array}$ & $\begin{array}{l}0,034 \text { a } \\
\text { (ND) }\end{array}$ & $\begin{array}{c}0,166 \mathrm{ab} \\
(4,5)\end{array}$ & $\begin{array}{l}3,881 \mathrm{e} \\
(66,7)\end{array}$ & $\begin{array}{l}4,732 \mathrm{~g} \\
(109,2)\end{array}$ \\
\hline \multicolumn{6}{|l|}{ Ray-grass } \\
\hline \multicolumn{6}{|l|}{ Témoin $=0,453 \mathrm{c}$} \\
\hline $60 \mathrm{t} / \mathrm{ha}$ & $0,172 \mathrm{a}$ & $0,165 \mathrm{a}$ & $0,270 \mathrm{~b}$ & $0,492 \mathrm{~cd}$ & $0,522 \mathrm{de}$ \\
\hline $240 \mathrm{t} / \mathrm{ha}$ & $0,130 \mathrm{a}$ & $0,144 \mathrm{a}$ & 0,176 a & $0,668 \mathrm{f}$ & $0,725 \mathrm{~g}$ \\
\hline
\end{tabular}

(*) Codification utilisée par Morel et al. (1986).

Nota : Les valeurs suivies de la même lettre pour une même culture ne sont pas significativement diffèrentes au seuil de 1 p. 100 (classement des moyennes par la méthode de Newman \& Keuls). Pour l'expérience “ laitue ", les valeurs entre parenthèses représentent les quantités d'azote exportées.

ND $=$ Non déterminé.

alors comparables à ceux obtenus sur le témoin recevant une fertilisation minérale.

On observe une très bonne corrélation entre les productions de ray-grass et de laitue. Pour les 2 doses communes (60 et $240 \mathrm{t} / \mathrm{ha}$ ), le coefficient de corrélation est égal à 0,986 (seuil 1 p. $100=0,76$ ).

La détermination de la teneur en azote total des laitues a permis de calculer les quantités d'azote exportées. Celles-ci augmentent avec l'âge du produit, c'està-dire lorsque les rapports $\mathrm{C} / \mathrm{N}$ et matière organique/ matière sèche diminuent. L'analyse statistique met en évidence une relation inverse entre l'azote exporté à la dose de $60 \mathrm{t} /$ ha et la teneur en carbone du produit $\left(r^{2}=0,914\right.$, seuil 5 p. $\left.100=0,878\right)$. Rappelons que BENISTANT (1978) a montré, sur les mêmes composts, que les produits âgés de moins de $180 \mathrm{j}$ immobilisaient l'azote minéral du sol alors qu'à partir de $180 \mathrm{j}$, on observe une minéralisation nette.

2. Expérience 2 : Comparaison de produits d'origines diverses pris à différents états d'évolution.

L'expérience précédente ayant montré que les résultats obtenus semblent indépendants de la nature de la plante utilisée, nous avons adopté le ray-grass pour la comparaison d'un grand nombre de produits différents.

Les produits issus de l'usine B (tabl. 2) montrent un effet dépressif maximal juste après prétraitement $(\mathrm{t}=2 \mathrm{j})$, puis cet effet diminue et s'annule après 4 mois de maturation ; il devient positif au-delà.
Le compost de l'usine $C$ provoque initialement un effet dépressif sévère ( 0 et 2 mois) qui s'atténue à 4 mois pour disparaître à partir de 6 mois d'évolution sur aire. Ces résultats sont tout à fait comparables à ceux obtenus précédemment avec le compost de l'usine $\mathrm{A}$.

Pour les produits de l'usine D, l'effet dépressif persiste plus longuement puisqu'il ne s'annule qu'à 8 mois. Ce ralentissement de la maturation est peutêtre à rapprocher de l'humidité excessive qui règnait dans les tas à partir de 4 mois.

Le compost de l'usine E provoque toujours un effet dépressif, quel que soit son âge. Là encore, cette anomalie est à mettre en relation avec les conditions d'évolution : le tas de compost, stocké en local couvert, s'est desséché progressivement, ce qui a pu ralentir les activités biologiques responsables de la « maturation ".

En ce qui concerne les ordures broyées, l'effet dépressif persiste plus de 8 mois ( $I$, tabl. 2).

Pour expliquer les diminutions observées de matière sèche récoltée en présence de composts jeunes, on ne peut exclure des phénomènes de phytotoxicité (JUSTE et al., 1980). Ceux-ci peuvent être dus à des éléments minéraux (Purves \& MACKENZIE, 1974 ; TroCME \& BONIFACE, 1977) susceptibles d'être lessivés ou insolubilisés lors du vieillissement du compost (JUSTE et al. 1980). Ils peuvent également être attribués à des composés organiques pouvant se former de façon transitoire lors du compostage (ZUCCONI et al., 1984). 
TABLEAU 2

Rendements en ray-grass ( $g$ M.S./pot) sur les mélanges sol + composts.

Rye-grass yields ( $g$ dry matter/pot) on soil-compost mixtures. Comparison with characteristics of composts.

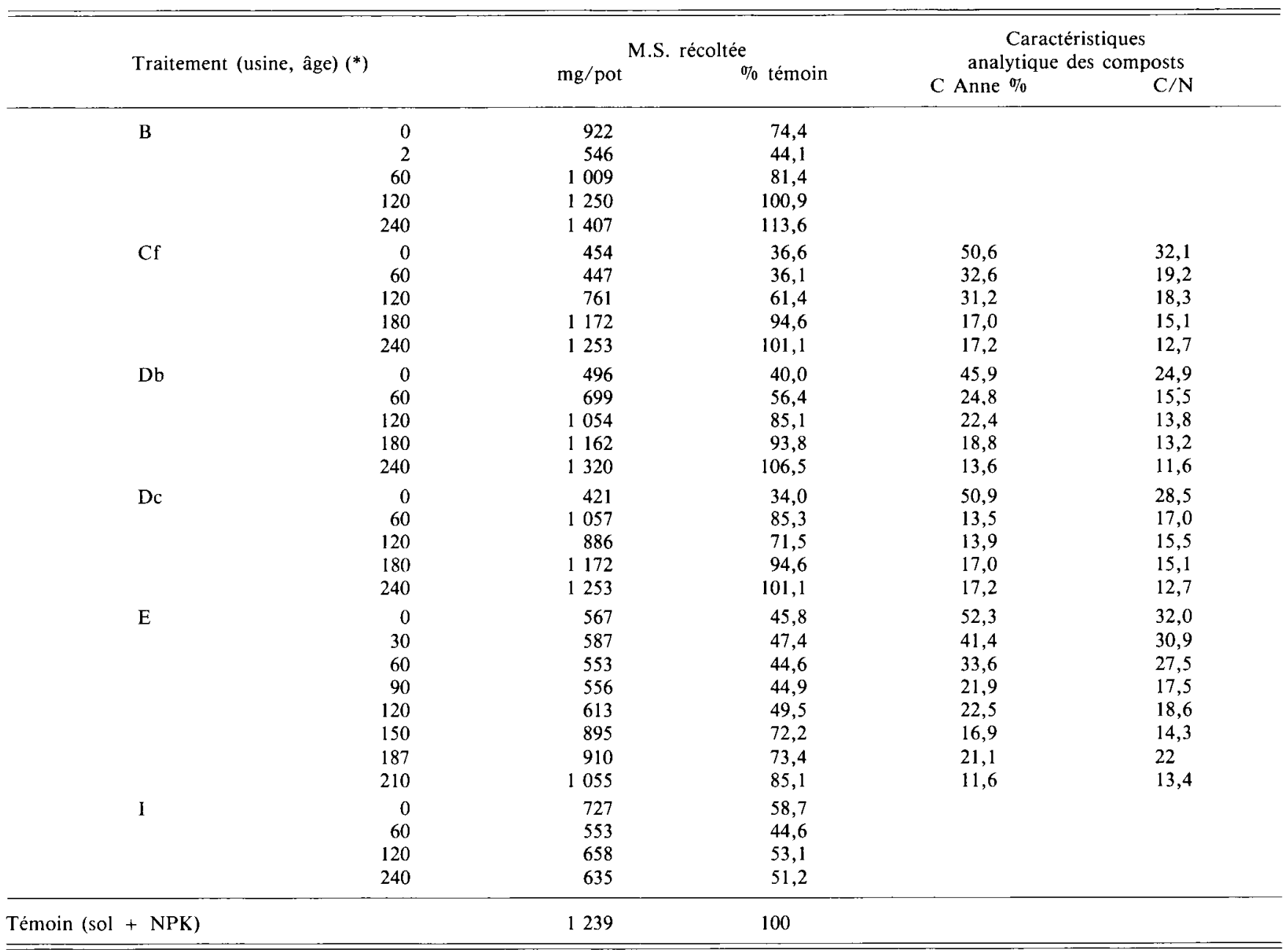

(*) Codification utilisée par MoRel et al. (1986).

Nota : Coefficient de variation moyen $=7$ p. 100.

Cependant, le ray-grass est une plante-test très connue pour sa résistance à ces facteurs, il est donc fort probable que l'effet «maturité » s'exerce avant tout à travers un effet-azote : les produits non-mûrs immobilisent de l'azote minéral, ce qui entraîne une diminution de la production végétale. Rappelons que CHAUSSOD et al. (1981), travaillant dans des conditions tout à fait comparables, ont montré qu'il y avait une relation parfaitement linéaire entre les quantités d'azote minéralisées à partir de boues résiduaires et les rendements en matière sèche de ray-grass.

\section{B. Comparaison des essais sur végétaux et des mesu- res respirométriques}

Les mesures en respirométrie électrolytique des consommations d'oxygène et les dégagements de $\mathrm{CO}_{2}$ sont reportés dans le tableau 3 pour les composts $\mathrm{Aa}$, $\mathrm{Cf}, \mathrm{Db}$ et $\mathrm{E}$. Les relations qui peuvent être établies entre la production de ray-grass et la respiration des composts exprimées de différentes façons, pour 18 produits venant de 3 usines différentes, dépendent en grande partie de la manière dont sont exprimées les mesures respiratoires (tabl. 4). C'est en rapportant la consommation d'oxygène à l'unité de matière sèche du produit que l'on obtient le plus fort coefficient de corrélation.

Cette dernière relation peut être assimilée à une courbe $\left(y=1,24 \mathrm{e}^{-0,075 \mathrm{x}}+0,41 ; \mathrm{r}^{2}=0,93\right)$ où y est la production de matière sèche et $\mathrm{x}$ la consommation d'oxygène (fig. 1).

La comparaison des productions de ray-grass dans un sol avec ou sans compost permettent de définir empiriquement 2 seuils d'activité respiratoire correspondant à 2 seuils de maturité du compost :

- Lorsque la consommation d'oxygène est inférieure à $7 \mathrm{mg} \mathrm{O}_{2}$ par $\mathrm{g}$ de matière sèche en $7 \mathrm{j}$ (17 p. 100 des composts étudiés), on peut être pratiquement certain que le compost est mûr, les effets sur végétaux étant par comparaison au témoin nuls ou positifs.

- Lorsque la consommation d'oxygène est supérieure à $15 \mathrm{mg} \mathrm{O}_{2}$ par $\mathrm{g}$ de matière sèche en $7 \mathrm{j}$ (55 p. 100 dés composts étudiés), on a la quasicertitude que le compost n'est pas mûr, la production 
TABLEAU 3

Données respirométriques et valeurs de "T.M.G. 21 " des différents composts étudiés. Respirometric data and " T.M.G. 21 》 values of the different composts studied.

\begin{tabular}{|c|c|c|c|c|}
\hline \multirow[b]{2}{*}{$\begin{array}{l}\text { Origine des } \\
\text { produits }(*)\end{array}$} & \multirow[b]{2}{*}{$\begin{array}{c}\text { Age } \\
\text { (en jours) }\end{array}$} & \multicolumn{2}{|c|}{$\begin{array}{c}\text { Respiration } \\
\text { (respirométrie électrolytique) }\end{array}$} & \multirow[b]{2}{*}{ «T.M.G. $21 »$} \\
\hline & & $\begin{array}{c}\text { Consommation } \mathrm{O}_{2} \\
\text { en } 7 \text { jours } \\
\text { (mg } \mathrm{O}_{2} / \mathrm{g} \mathrm{M.S.)}\end{array}$ & $\begin{array}{c}\text { Production } \mathrm{CO}_{2} \\
\text { en } 7 \text { jours } \\
\text { (mg CO } \mathrm{CO}_{2} / \mathrm{g} \mathrm{M.S.)}\end{array}$ & \\
\hline \multirow[t]{4}{*}{$\mathrm{Aa}$} & 0 & 58,1 & - & 6,49 \\
\hline & 60 & 45,6 & - & 5,77 \\
\hline & 120 & 30,7 & - & 3,95 \\
\hline & 240 & 5,7 & - & 2,65 \\
\hline \multirow[t]{5}{*}{$\mathrm{Cf}$} & 0 & 36,8 & 42,8 & - \\
\hline & 60 & 32,1 & 34,8 & - \\
\hline & 120 & 17,5 & 29,9 & - \\
\hline & 187 & 7,0 & 7,7 & - \\
\hline & 210 & 3,3 & 4,1 & - \\
\hline \multirow[t]{5}{*}{$\mathrm{Db}$} & 0 & 41,4 & 46,1 & 4,39 \\
\hline & 60 & 24,3 & 27,0 & 4,13 \\
\hline & 120 & 10,3 & 15,0 & 2,74 \\
\hline & 180 & 9,2 & 12,6 & - \\
\hline & 240 & 4,4 & 5,0 & 2,17 \\
\hline \multirow[t]{8}{*}{$E$} & 0 & 22,5 & 25,0 & - \\
\hline & 30 & 37,5 & 44,8 & - \\
\hline & 60 & 29,0 & 29,2 & - \\
\hline & 90 & 21,8 & 25,9 & - \\
\hline & 120 & 24,1 & 23,9 & - \\
\hline & 150 & 8,9 & 11,0 & - \\
\hline & 187 & 10,3 & 10,8 & - \\
\hline & 210 & 9,2 & 10,0 & - \\
\hline
\end{tabular}

$\left({ }^{*}\right)$ Codification utilisée par Morel et al. (1986).

Nota : - absence de mesure.

Coefficients de variation inférieurs à 5 p. 100 .

TABLEAU 4

Coefficients de corrélation entre la production de matière sèche et l'activité respiratoire exprimée de différentes façons. (Composts $C f$. Db et E) (*).

Correlation coefficient between dry matter production and respiratory activity expressed in different ways. (Composts $C f, D b$ and $E)(*)$.

\begin{tabular}{|c|c|}
\hline Relation étudiée & $\begin{array}{l}\text { Coefficient } \\
\text { de corrélation }\end{array}$ \\
\hline $\begin{array}{l}\text { - Production de ray-grass/dégagement } \mathrm{du} \mathrm{CO}_{2} / \mathrm{g} \text { de matière sèche } \\
\text { - Production de ray-grass/consommation } \mathrm{d}^{\prime} \mathrm{O}_{2} / \mathrm{g} \text { de matière seche } \\
\text { - Production de ray-grass/consommation } \mathrm{d}^{\prime} \mathrm{O}_{2} / \mathrm{g} \text { d'azote total } \\
\text { - Production de ray-grass/consommation } \mathrm{d}^{\prime} \mathrm{O}_{2} / \mathrm{g} \text { de carbone Anne } \\
\text { - Production de ray-grass/consommation } \mathrm{d}^{\prime} \mathrm{O}_{2} / \mathrm{g} \text { de matière volatile }\end{array}$ & $\begin{array}{l}-0,885 \\
-0,905 \\
-0,893 \\
-0,687 \\
-0,886\end{array}$ \\
\hline
\end{tabular}

(*) Codification utilisée par Morel. et al. (1986).

Nota : Seuil de signification à 1 p. $100=0,589$.

de ray-grass dans le sol avec compost étant systématiquement inférieure à celle du témoin.

Entre ces 2 valeurs, il semble difficile de se prononcer, divers produits ayant une respiration identique pouvant entraîner des effets différents. Bien entendu, la définition des seuils de maturité de cette étude reste liée aux conditions expérimentales, notamment le type de sol utilisé, la plante-test et la dose de compost choisie.

\section{Méthodes simples d'appréciation de la maturité des composts urbains}

\section{Test respirométrique simplifié}

L'activité respiratoire de 3 produits de maturités différentes mesurée par le test respirométrique simplifié permet de déterminer facilement la phase de latence et la partie de la courbe utilisable pour le cal- 


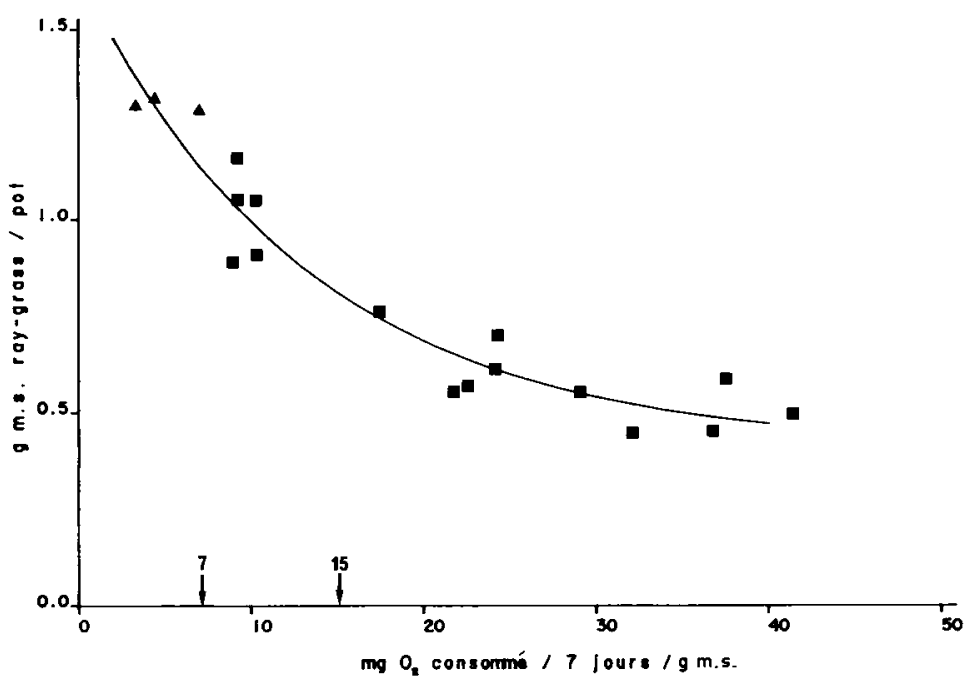

Figure 1

Relations entre consommation d'oxygène et production de ray-grass (ム production ray-grass > témoin sol sans apport de compost). (ם production ray-grass $\leqslant$ témoin sol sans apport de compost).
Relationships between oxygen consumption and rye-grass yield (A rye-grass yield > control soil without compost enrichment). (ם rye-grass yield $\leqslant$ control soil without compost enrichment).

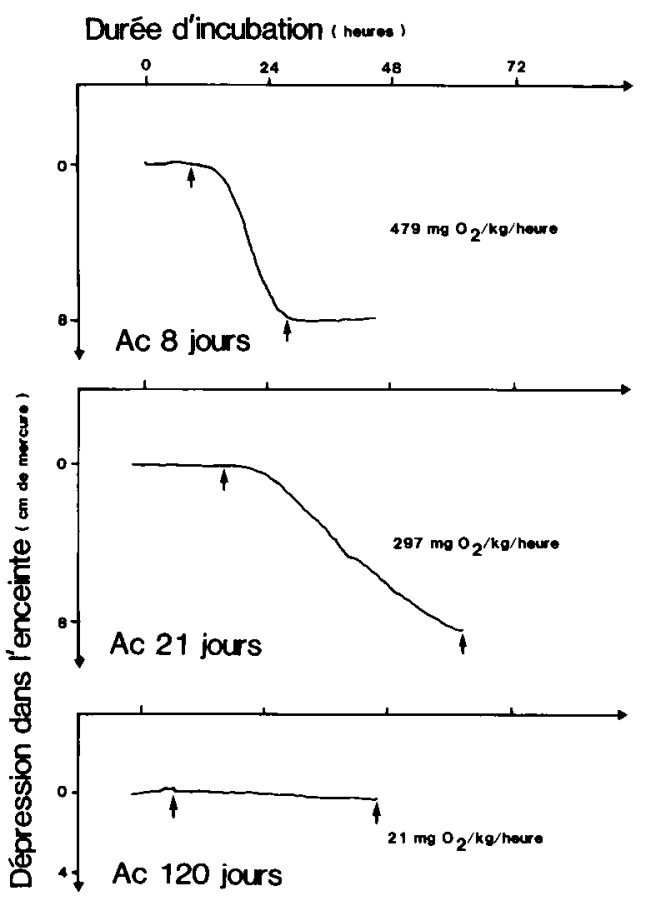

Figure 2

Diagrammes obtenus pour 3 produits à l'aide du test simple (l'intervalle entre les flèches détermine le temps de consommation d'oxygène pris en considération).

Le chiffre inscrit dans chaque diagramme correspond à la consommation d'oxygène calculée avec la courbe et exprimée en $\mathrm{mg}$ par $\mathrm{kg}$ de produit sec et par heure.

Graphs of 3 products obtained in the simple test.

(The space between the two marks determines the oxygen consumption time considered.

The value - written on each graph - corresponds to the oxygen consumption calculated with the curve and expressed in $\mathrm{mg} / \mathrm{kg} d r y$ matter/h.

cul de la consommation d' $\mathrm{O}_{2}$ du compost (fig. 2). Les 2 méthodes respirométriques (électrolytique et simplifiée) donnent des mesures bien corrélées entre elles (pour 9 échantillons : $\mathrm{r}=0,96$; seuil à 1 p. $100=$
TABLEAU 5

Valeurs de consommations d'oxygène obtenues avec le test simple et avec la méthode de respirométrie électrolytique.

Oxygen consumption values obtained with the simple method and the electrolytic respirometric method.

\begin{tabular}{lccc}
\hline \multirow{2}{*}{$\begin{array}{c}\text { Origine } \\
\text { des } \\
\text { composts }\left(^{*}\right)\end{array}$} & $\begin{array}{c}\text { Date } \\
\text { (jours) }\end{array}$ & $\begin{array}{c}\text { Consommation } \mathrm{O}_{2} \\
\text { (test respirométrique } \\
\text { simple) }\end{array}$ & $\begin{array}{c}\text { Consommation } \mathrm{O}_{2} \\
\text { (respirométrie } \\
\text { électrolytique) }\end{array}$ \\
\hline $\mathrm{na}$ & 0 & 185 & 286,2 \\
\cline { 2 - 4 } & 45 & 29 & 28,1 \\
\hline & 75 & 22 & 32,9 \\
\hline $\mathrm{Cf}$ & 240 & 23 & 26,4 \\
\cline { 2 - 4 } & 187 & 251 & 307,4 \\
\cline { 2 - 4 } & 210 & 50 & 41,9 \\
\hline $\mathrm{E}$ & 180 & 21 & 61,5 \\
\hline & 210 & 81 & 54,9 \\
\hline
\end{tabular}

(*) Codification utilisée par MoRel. et al. (1986).

Nota: Tous les produits ont été conservés à l'état frais à $4{ }^{\circ} \mathrm{C}$ après le prélèvement.

Les valeurs sont exprimées en $\mathrm{mg} \mathrm{O}_{2} / \mathrm{kg}$ M.S. $/ \mathrm{h}$.

0.79) (tabl. 5). Sous réserve d'un plus large échantillonnage, l'utilisation des mêmes seuils de maturité définis précédemment paraît a priori possible. Cette méthode devrait donc pouvoir être utilisée dans la pratique. Toutefois, certaines précautions sont à prendre :

- la possibilité de phase de latence allant au-delà de $3 \mathrm{j}$ implique de poursuivre les observations jusqu'à $5 \mathrm{j}$ pour conclure à la maturité du compost. Si l'activité respiratoire ne se manifeste qu'au $4^{\mathrm{e}}$ ou $5^{\mathrm{e}} \mathrm{j}$, il faut alors continuer jusqu'à 6 ou $7 j$ pour obtenir une mesure correcte ; 
- il apparaît indispensable de standardiser les conditions d'utilisation, notamment en ce qui concerne l'humidité qui, lors du prélèvement, peut être excessivement variable. Des essais complémentaires (NICOLARDOT et al., 1982) ont montré que l'on peut facilement déterminer une capacité de rétention pour l'eau d'un compost très grossier à l'aide d'une essoreuse ménagère. Le niveau d'humidité résiduelle d'un échantillon soumis à environ $50 \overrightarrow{\mathrm{g}}$ pendant quelques minutes convient très bien pour l'incubation ;

- la mesure respiratoire ainsi réalisée sur un large échantillonnage de composts et d'ordures broyées est apparue satisfaisante, l'activité respiratoire décroissant régulièrement avec l'âge du matériau (GERMON et al., 1980). Il est possible, dans certains cas, de ne pas observer d'activité respiratoire mesurable : tel fut le cas d'un compost en sortie d'hygiénisateur et un autre âgé de 2 mois. Il s'agit ici de la présence probable dans le compost de produits toxiques pour les microorganismes.

\section{Caractérisation de composés biodégradables}

Le «T.M.G. 21 » établi à partir des résultats de la dégradation de composts dans un seul type de sol est corrélé significativement avec différents paramètres mesurés sur un ensemble de 73 composts (MOREL \& JACQUIN, 1979) (tabl. 6). Les variables les mieux corrélées sont l'âge du compost, la teneur en carbone organique total (C.O.T.) et la teneur en polysaccharides hydrosolubles (GS). L'analyse en régression multiple a alors permis d'établir la relation suivante :

T.M.G. $21=3,166-0,011$ âge $+0,059$ C.O.T. $+0,832$ Ln GS avec $r^{2}=0,730$ et $\sigma^{2}=0,967$.

L'âge est exprimé en jours, le carbone organique total (C.O.T.) et les glucides hydrosolubles (GS) en pourcentage de la matière sèche. Connaissant l'âge d'un compost et ses teneurs en certains composés carbonés faciles à doser, il apparaît possible d'en prévoir la vitesse de minéralisation lors de son enfouissement dans le sol sans avoir à recourir à une expérimentation complexe.

Par ailleurs, les composts $\mathrm{Aa}$ et $\mathrm{Db}$ ont servi à établir une relation entre le «T.M.G. 21 » et la consommation d'oxygène. La corrélation est hautement significative $(r=0,97$; seuil à 1 p. $100: 0,83)$. Elle permet de faire correspondre 2 valeurs de «T.M.G. 21 » aux 2 valeurs seuils de consommation d'oxygène définies précédemment : ainsi, un compost pourrait être considéré comme mûr quand son «T.M.G. 21 » descend en dessous de 2,4 et n'est certainement pas mûr lorsqu'il dépasse 2,8 .
La définition de ces seuils permet de situer un compost sur une "échelle de maturité ". Cette méthode nécessite de connaître avec précision l'âge du compost et de disposer d'un laboratoire où l'on puisse doser carbone organique et polysaccharides hydrosolubles. Cette approche est complémentaire de la respirométrie directe. Elle permet de s'affranchir des problèmes dus à des composts ne respirant pas en raison de la présence de substances toxiques pour la microflore et contenant encore de fortes quantités de composés fermentescibles.

\section{CONCLUSIONS}

Dans la présente étude, nous avons cherché à caractériser les composts en vue d'une utilisation en grande culture, en nous attachant aux effets à court terme sur la croissance des plantes. Des composts jeunes provoquent fréquemment un effet dépressif sur la végétation ; cet effet s'atténue et disparaît avec les composts âgés. Ces observations conduisent à définir un seuil de maturité qui est le stade d'évolution à partir duquel le compost ajouté au sol ne provoque plus d'effet dépressif sur la végétation.

Si l'on excepte, dans un premier temps, les problèmes de phytotoxicité, on peut en déduire une définition du "seuil de maturité » comme étant l'état d'évolution d'un compost qui n'entraîne plus d'immobilisation microbienne de l'azote.

Pour caractériser plus rapidement ce stade d'évolution, la voie la plus simple apparaît être d'évaluer le carbone facilement assimilable par les microorganismes car c'est la disponibilité en carbone qui détermine leurs besoins en azote. L'importante évolution quantitative et qualitative du carbone organique observée par MoREL et al. (1986) a permis d'envisager des tests de maturité dans 2 directions différentes, tests qui ont été calibrés à l'aide des expérimentations sur plantes :

- en vérifiant le bien-fondé de la méthode respirométrique pour apprécier la maturité des composts, nous avons défini dans nos conditions expérimentales 2 seuils de maturité : pour une consommation d'oxygène inférieure à $7 \mathrm{mg} / \mathrm{g}$ de matière sèche en $7 \mathrm{j}$, on peut être pratiquement certain que le compost est mûr ; au-delà de $15 \mathrm{mg} / \mathrm{g}$ de matière sèche en $7 \mathrm{j}$, on est sûr qu'il ne l'est pas. A la suite de ces expériences, nous avons pu proposer une méthode respirométrique, simplifiée facilement utilisable en usine donnant les résultats au bout de 3 à $7 \mathrm{j}$;

\section{TABLEAU 6}

Coefficients de corrélation (r) entre le taux de minéralisation globale après 21 jours d'incubation et quelques caractéristiques du compost. Correlation coefficients (r) between the total mineralisation rate after a 21-day incubation period and some characteristics of compost.

\begin{tabular}{|c|c|c|c|c|c|}
\hline $\begin{array}{l}\text { Age du } \\
\text { compost }\end{array}$ & hydrosolubles & hydrolysables & $\begin{array}{c}\text { Carbone organique } \\
\text { total }\end{array}$ & Azote total & $\mathrm{C} / \mathrm{N}$ \\
\hline 0,82 & 0,42 & 0,70 & 0,67 & $-0,014$ & 0,55 \\
\hline
\end{tabular}

Nota: Seuil de signification à 1 p. $100=0,30$.

Les mesures de sucres sont transformées en données logarithmiques. 
- la détermination d'un indice de maturité «T.M.G. 21 » à partir de déterminations chimiques est une approche complémentaire intéressante permettant de s'assurer que la non-respiration est bien due à l'épuisement des sucres utilisables.

La combinaison de ces 2 méthodes apparaît intéressante dans le cadre du contrôle de la qualité des composts et du bon déroulement des processus de fermentation. Ces 2 indices, taux de respiration et «T.M.G. $21 »$, pourraient donc être utilisés comme critères de qualité de produits mis sur le marché.

Reçu le 20 avril 1985. Accepté le Il juin 1986.

\section{REMERCIEMENTS}

Nous tenons à remercier J. P. MAItre (ENITA Dijon) et ses étudiants pour leur participation à l'expérimentation sur laitue.

Ces travaux ont bénéficié d'aides du Ministère de l'Environnement.

\section{RÉFÉRENCES BIBLIOGRAPHIQUES}

Afnor, 1981. Amendements organiques. Dénominations et spécifications (Norme NF U 44-051).

Ahrens E., Farkasdi G., Ibrahim I., 1965. Quelques critères servant à caractériser la putréfaction des gadoues. Bull. Inf. G.I.R.O.M., Zurich, 24, 17-24.

Allenspach H., 1969. Détermination du degré de maturité du compost de gadoues. $1^{\text {re }}$ Partie : Mesure de la diminution d'oxygène. Bull. Inf. G.I.R.O.M., Zurich, 35, 330-342.

Benistant D., 1978. Caractérisation de la maturité des composts d'ordures ménagères. Mémoire de fin d'études E.N.I.T.A., Dijon, $40 \mathrm{p}$.

Bidlingmaier W., 1984. Quality testing of waste sludge composts, 28 p. Commun. in E.E.C. seminar on composting agricultural and other wastes. Oxford, march 1984, $320 \mathrm{p}$.

Chaussod R., Germon J. C., Catroux G., 1981. Essai de détermination au laboratoire de l'aptitude à la minéralisation d'azote des boues résiduaires urbaines. C. R. Acad. Agric. Fr., 67, 762-771.

Chrometzka P., 1968a. Der Einfluss von vorschiedenen Reifestadien des Kompostes auf das Wachstum von Bodenpilzen. Organischer Landbau, 11, 4/5, 63-64.

Chrometzka P., 1968b. Détermination de la consommation d'oxygène des composts en voie de maturation. Bull. Inf. G.I.R.O.M., Zurich, 33, 253-256.

Colin F., 1977. Mise au point d'une méthode de détermination de l'ATP dans les composts et application à leur caractérisation. In Ministère de l'Environnement et du Cadre de Vie : "Sols et déchets solides ", 11, 355-360.

Germon J. C., Verguet A., 1974. Description d'un respiromètre électrolytique utilisé pour étudier la biodégradabilité d'effluents apportés au sol. Ann. Agron., 25, 195-205.

Germon J. C., Nicolardot B., Catroux G., 1980. Mise au point d'un test rapide de détermination de la maturité des composts. Convention d'étude 79-509. Ministère de l'Environnement et du Cadre de Vie, 8 p.

Hertelendy K., 1974. Paper chromatography, a quick method to determine the degree of humification of refuse compost. I.R.C.W.D. News, W.H.O., Dübendorf (CH), 7, 1-5.

Hirsheydt A. (Von), 1977. Détermination du terme « maturité » en matière de compost de gadoues. I.S.W.A. Inf. Bull., EAWAG, Dübendorf $(\mathrm{CH}), 21,5$.

Juste C., Solda P., 1977. Etude des possibilités d'utilisation des composts d'ordures ménagères comme supports de cultures maraîchères. In Ministère de l'Environnement et du Cadre de Vie : « Sols et déchets solides ", 11, 389-396.

Juste C., Solda P., Dureau P., 1980. Mise au point de tesis agronomiques légers permettant de déterminer simultanément la phytotoxicité globale des composts d'ordures ménagères et leur degré de maturation. Convention d'étude 77-147. Ministère de l'Environnement et du Cadre de Vie, 19 p.
Lossin R. D., 1970. Compost studies. Part I. Compost Sci., 11 (6), 16-17.

Lossin R. D., 1971. Compost studies. Part III. Disposing of animal wastes. Measurement of the chemical oxygen demand of compost. Compost Sci., 12 (2), 31-32.

Moller F., 1968. Potentiel d'oxydo-réduction et qualité hygiénique des composts de résidus d'agglomérations. Bull. Inf. G.I.R.O.M., Zurich, 32, 228-233.

Morel J. L., 1982. L'évolution de la maturité des composts urbains par une méthode colorimétrique. Compost Inf., 10, 4-8.

Morel J. L., Jacquin F., 1979. An approach for the determination of urban compost maturity. Humus et Planta, 7, 321-326.

Morel J. L., Guckert A., Nicolardot B., Benistant D., Catroux G., Germon J. C., 1986. Etude de l'évolution des caractéristiques physico-chimiques et de la stabilité biologique des ordures ménagères au cours du compostage. Agronomie, 6 (8), 693-701.

Nicolardot B., Germon J. C., Chaussod R., Catroux G., 1982. Une technique simple pour déterminer la maturité des composts urbains. Compost Inf., 10, 2-4.

Niese G., 1969. Die Bestimmung des biologischen Sauerstoffbedarfs von Komposten mit Hilfe des Warburg-Apparatur. Biol. sol., 10, 28-30.

Obrist W., 1965. Activité enzymatique et processus de décomposition se déroulant dans les gadoues en putréfaction; nouvelle méthode proposée pour la détermination du degré de putréfaction. Bull. Inf. G.I.R.O.M., Zurich, 24, 29-37.

Pommel B., Juste C., 1977. La valorisation agricole de déchets. I. Le compost urbain. S.E.I., C.N.R.A., 75 p.

Purves D., Mackenzie E., 1974. Phytotoxicity due to boron in municipal compost. Plant Soil, 40, 231-235.

Rolle G., Orsanic B., 1964. Nouvelle méthode pour la détermination des substances organiques décomposables et résistantes présentes dans les gadoues et dans les composts de gadoues. Bull. Inf. G.I.R.O.M., Zurich, 21, 3-12.

Spohn E., 1968. Wie reif ist ein Kompost. Städtehygiene, 6, 116120.

Spohn E., 1978. Determination of compost maturity. Compost Sci., May/June, 26-27.

Spohn E., Kneer F., 1968. Über die Atmung von Kompost. Organischer Landbau, 11, 4/5, 68-71.

Trocmé S., Boniface R., 1977. Risques de toxicité dus à des teneurs élevées en bore des boues résiduaires et des composts urbains. In Ministère de l'Environnement et du Cadre de Vie. "Sols et déchets solides ", 11, 397-399.

Zucconi F., Monaco A., Forte M., 1984. Phytotoxins during the stabilization of organic matter, 73-86. In E.E.C. Seminar on composting agricultural and other wastes, Oxford, march 1984, $320 \mathrm{p}$. 